\title{
Involvement of RhoA/ROCK in myocardial fibrosis in a rat model of type 2 diabetes
}

\author{
Hong ZHOU ${ }^{1}$, Yong-jun $\mathrm{LI}^{2, *}$, Mian WANG ${ }^{1}$, Li-hui ZHANG ${ }^{1}$, Bing-yan $\mathrm{GUO}^{2}$, Zhan-sheng ZHAO ${ }^{1}$, Feng-ling MENG ${ }^{1}$, \\ Yong-gui $D_{E N G}{ }^{1}$, Rui-ying WANG ${ }^{1}$ \\ Department of ${ }^{1}$ Endocrinology and ${ }^{2}$ Cardiology, the Second Hospital of Hebei Medical University, Shijiazhuang 050000, China
}

\begin{abstract}
Aim: To investigate whether activation of RhoA/Rho kinase (ROCK) is involved in myocardial fibrosis in diabetic hearts.
Methods: A rat model of type 2 diabetes was established using high fat diet combined with streptozotocin ( $30 \mathrm{mg} / \mathrm{kg}$, ip). Animals were randomly divided into 3 groups: control rats, untreated diabetic rats that received vehicle and treated diabetic rats that received Rhokinase inhibitor fasudil hydrochloride hydrate $\left(10 \mathrm{mg}_{\mathrm{kg}} \cdot \mathrm{d}^{-1}\right.$, ip, for 14 weeks). Cardiac contractile function was evaluated in vivo. The morphological features of cardiac fibrosis were observed using immunohistochemistry and TEM. The mRNA expression of JNK, TGF $\beta 1$, type-I, and type-III procollagen was assessed with RT-PCR. The phosphorylation of MYPT1, JNK and Smad2/3, as well as the protein levels of TGFB1 and c-Jun, were evaluated using Western blotting.

Results: In untreated diabetic rats, myocardial fibrosis was developed and the heart contractility was significantly reduced as compared to the control rats. In the hearts of untreated diabetic rats, the mRNA expression level and activity of JNK were upregulated; the expression of TGFB1 and phosphorylation of Smad2/3 were increased. In the hearts of treated diabetic rat, activation of JNK and TGFB/Smad was significantly decreased, myocardial fibrosis was reduced, and cardiac contractile function improved.

Conclusion: The data suggest that fasudil hydrochloride hydrate ameliorates myocardial fibrosis in rats with type 2 diabetes at least in part through inhibiting the JNK and TGF $\beta /$ Smad pathways. Inhibition of RhoA/ROCK may be a novel therapeutic target for prevention of diabetic cardiomyopathy.
\end{abstract}

Keywords: diabetes; myocardial fibrosis; Rho kinase; c-Jun $\mathrm{NH}_{2}$-terminal kinase; transforming growth factor- $\beta$

Acta Pharmacologica Sinica (2011) 32: 999-1008; doi: 10.1038/aps.2011.54; published online 11 Jul 2011

\section{Introduction}

Diabetes mellitus (DM) has emerged as a major threat to the health of humans throughout the world. Long-term DM results in the development of diabetic cardiomyopathy, which is characterized histologically by myocardial fibrosis. DMinduced cardiac remodeling may participate in diastolic and systolic dysfunction, leading to congestive heart failure. Moreover, it can occur during the early stages of $\mathrm{DM}^{[1,2]}$, and is closely related to a high prevalence of mortality from cardiovascular events in DM. However, specific drugs for the prevention or treatment of diabetic cardiomyopathy are not available. Treatment of heart failure is empirical because a class of agent that favorably affects outcomes has not been discovered. Thus, a more thorough understanding of the pathogenesis of diabetic cardiomyopathy and the development of new phar-

\footnotetext{
* To whom correspondence should be addressed.

E-mail lyjbs2009@yeah.net

Received 2010-01-23 Accepted 2011-04-13
}

macologic treatments are needed.

RhoA is a member of the Ras superfamily of GTP-binding proteins. Its downstream effector, Rho-kinases (ROCK), regulates various biological functions in cells, including the adherence, migration, proliferation, apoptosis and gene expression of cells ${ }^{[3,4]}$. Also, the RhoA/ROCK pathway plays an important pathophysiological part in hypertension ${ }^{[5]}$ and stroke ${ }^{[6]}$. There is growing evidence that RhoA/ROCK signaling mediates the glomerular matrix accumulation of proteins in diabetic nephropathy. The ROCK inhibitor fasudil can suppress renal fibrosis and can reduce proteinuria ${ }^{[7,8]}$. The inhibition of ROCK attenuates cardiac fibrosis in angiotensin II-induced cardiac hypertrophy in vivo ${ }^{[9,10]}$. Mitogen-activated protein kinases (MAPKs) are important mediators of signal transduction from the cell surface to the nucleus. c-Jun $\mathrm{NH}_{2}$-terminal kinase (JNK) is one of the major members of MAPKs, and JNK activation is also implicated in cardiac fibrosis ${ }^{[11,12]}$. Recent studies show that there is signal crosstalk between RhoA/ ROCK and JNK pathways ${ }^{[13]}$. The pro-sclerotic growth factor, 
transforming growth factor $\beta$ (TGF $\beta$ ), plays a major part in the fibrosis and dysfunction of organs ${ }^{[14,15]}$. Hence, the blockade of its expression and action represents an important therapeutic target.

A high-fat diet with injection of low-dose streptozotocin (STZ) for the induction of type $2 \mathrm{DM}$ in rats has been found to be a good model for DM research ${ }^{[16,17]}$. This type of model replicates the natural history and metabolic characteristics of type $2 \mathrm{DM}$ in humans.

The purpose of the present study was to understand the roles of RhoA/ROCK, JNK, and TGF $\beta /$ Smad pathways in DM-induced cardiac remodeling. We also wished to ascertain the effects of fasudil on myocardial fibrosis in rats with type $2 \mathrm{DM}$, with the objective of providing a novel target for the treatment of diabetic cardiomyopathy.

\section{Materials and methods}

The experimental protocol was approved by the Ethics Review Committee for Animal Experimentation of Hebei Medical University (Shijiazhuang, China).

\section{Experimental animals}

Female Sprague-Dawley (SD) rats (initially weighing 180-200 g) were procured from the Laboratory Animal Centre of Hebei Medical University. Rats were housed in standard cages (4 rats/cage) and maintained under a controlled room temperature $\left(22 \pm 2{ }^{\circ} \mathrm{C}\right)$ and humidity $(55 \% \pm 5 \%)$ with a 12 -h: 12 -h light-dark cycle. After acclimatization, rats were randomly assigned to receive a standardized diet (18\% fat, $25 \%$ protein, and $57 \%$ carbohydrate) as the normal control group or a high-fat diet (HFD, $40 \%$ fat, $13 \%$ protein, and $47 \%$ carbohydrate) for 6 weeks. At the end of 6 weeks, as previously described $^{[16,17]}$, control rats were injected (ip) with vehicle [0.1 $\mathrm{mol} / \mathrm{L}$ citric acid buffer ( $\mathrm{pH} 4.5)]$ once and given a standard diet sequentially. HFD rats were injected (ip) with low-dose (30 mg/kg) of STZ (Sigma-Aldrich, St Louis, MO, USA) and given a high-fat diet sequentially. Blood glucose (BG) was measured in whole blood collected from the tail vein by a portable glucometer (Accu-Chek Active, Roche Diagnostics Limited, Mannheim, Germany). Body weight (BW) and BG levels were recorded every week. Rats with BG levels above 16.7 $\mathrm{mmol} / \mathrm{L}$ and that were stable for 4 weeks were considered to be diabetic. At week 10, hyperinsulinemic-euglycemic clamp techniques were performed, and fasting insulin (FINS), fasting blood glucose (FBG), total cholesterol (TC) and triglyceride (TG) were measured.

Control rats remained on a standard diet and were treated with ip injection of sterile vehicle every day. Diabetic rats were randomly divided into two groups: (i) treated diabetic rats maintained on a high-fat diet and received fasudil hydrochloride hydrate (hereafter termed "fasudil"; $10 \mathrm{mg} \cdot \mathrm{kg}^{-1} \cdot \mathrm{d}^{-1}$; ip) (Tianjin Hongri Company, Tianjin, China) every day for 14 weeks; and (ii) untreated diabetic rats treated with injection (ip) of sterile vehicle every day for 14 weeks. Systolic arterial blood pressure (SABP) was measured monthly by tail-cuff micro-photoelectric plethysmography. At week 24 , cardiac contractile function was evaluated. Fasting plasma was collected for further measurement of FINS, FBG, TG, TC, and glycosylated hemoglobin (HbA1c). The homeostasis model assessment for insulin resistance (HOMA-IR) was calculated as FBG $\times$ FINS/22.5 and was used assessing insulin resistance ${ }^{[18]}$.

The rats were killed, the hearts were harvested and dried weights were recorded. A piece of tissue from the left ventricle was fixed in $4 \%$ paraformaldehyde and embedded in paraffin for histological examination. The remaining tissues were snap-frozen in liquid nitrogen and stored at $-80{ }^{\circ} \mathrm{C}$ until processing for extraction of mRNA and protein.

\section{Hyperinsulinemic-euglycemic clamp techniques}

As previously described ${ }^{[19]}$, rats were fasted for 12-14 h overnight before the clamp study began. Rats were anesthetized by an injection of pentobarbital sodium $(48 \mathrm{mg} / \mathrm{kg}$; ip). The right jugular vein was cannulated for the infusion of glucose (using separate catheters) and insulin; the left carotid artery was cannulated for blood sampling. The glucose solution and insulin solution were stored respectively in two digital syringe pumps, and jointed by a " $Y$ " connector to the jugular catheter. Insulin (Novolin R, Novo Nordisk Pharmaceuticals, Bagsvaerd, Denmark) was infused at $11 \mathrm{mU} \cdot \mathrm{kg}^{-1} \cdot \mathrm{min}^{-1}$ (insulin concentration $100 \mathrm{mU} / \mathrm{mL}$ ) through the jugular vein catheter and BG levels serially monitored using a glucometer. The insulin infusion rate was permanent. When BG levels were within euglycemic levels $(5.0 \pm 0.5 \mathrm{mmol} / \mathrm{L})$, infusion of $5 \%$ glucose was started. The glucose infusion rate (GIR) was adjusted every 5-10 min (as needed) to maintain the BG level at a euglycemic level. Steady state was achieved by $60 \mathrm{~min}$ and maintained for $30 \mathrm{~min}$. The mean GIR was calculated based on GIR readings corresponding to the last six samplings.

\section{Measurement of cardiac contractile function}

The cardiac contractile function in vivo was measured at week 24. Rats were anesthetized with pentobarbital sodium (48 $\mathrm{mg} / \mathrm{kg}$; ip). A miniaturized combined catheter-micromanometer was inserted in the right common carotid artery to measure the aortic blood pressure. It was then advanced in the left ventricle to measure left ventricular (LV) pressure-volume loops. Heart rate (HR), left ventricular systolic pressure (LVSP), left ventricular end-diastolic pressure (LVEDP) and maximum change velocity of left ventricular pressure in the isovolumic contraction or relaxation period $\left( \pm \mathrm{d} p / \mathrm{d} t_{\max }\right)$ were measured by a BL-420E Data Acquisition \& Analysis System (TME Technology Company Limited, Chengdu, China).

\section{Studies using transmission electron microscopy (TEM) and immunohistochemistry}

Specimens were fixed in $4 \%$ paraformaldehyde containing $0.25 \%$ glutaraldehyde and $4.5 \%$ sucrose. Ultra-thin sections obtained from the embedded blocks were examined using a TEM (H-7500; Hitachi, Tokyo, Japan). Masson staining for collagen and streptavidin-biotin-peroxidase complex (SABC) immunohistochemistry staining for type-I collagen and typeIII collagen (rabbit antibodies from Santa Cruz Biotechnol- 
ogy, Santa Cruz, CA, USA) were undertaken after 4- $\mu$ m thick sections were obtained from the paraffin-embedded cardiac samples using previously described methods ${ }^{[20]}$. Results were expressed as the mean percentage area of 10 random fields for each section.

\section{Isolation of RNA and reverse transcription-polymerase chain} reaction (RT-PCR)

Total mRNA from heart tissues was extracted using TRIzol reagent according to the manufacturer's protocol. The purity of isolated RNA was identified by ultraviolet spectrometry. Single-strand cDNA was synthesized by reverse transcription using oligo (dT) primer and M-MLV reverse transcriptase (Promega, Fitchburg, WI, USA), which was used as a template in the subsequent PCR analysis. Forward and reverse primers were synthesized and purified by Shanghai Sangon Biotechnology, Incorporated (Shanghai, China). The primers were JNK: forward 5'-TCAGAATCCGAACGAGACAAAAT-3' and reverse 5'-AAGCCAGAGTCCTTCACAGACAA-3'; 469 bp. TGF $\beta 1$ : forward 5'-CCAAGGAGACGGAATACAGG-3' and reverse 5'-GTGTTGGTTGTAGAGGGC AAG-3'; 411 bp. For type-I procollagen: forward 5'-TTCACCTACAGCACGCTTGT-3' and reverse 5'-TTGGGATGGAGGGAGTTTAC-3'; 196 bp. For type-III procollagen: forward 5'-TTGAATATCAAACACGCAAGGC-3' and reverse 5'-GGTCACTTTCACTGGTTGACGA-3'; 201 bp. For GAPDH: forward 5'-GATGGGTGTGAACCACGAGAAA-3' and reverse 5'-ACGGATACATTGGGGGTAGGAA-3'; 330 bp. The JNK amplification condition was pre-denaturation at $94^{\circ} \mathrm{C}$ for $5 \mathrm{~min}$, denaturation at 94 ${ }^{\circ} \mathrm{C}$ for $30 \mathrm{~s}$, annealing at $61^{\circ} \mathrm{C}$ for $30 \mathrm{~s}$, and extension at $72{ }^{\circ} \mathrm{C}$ for $30 \mathrm{~s}$, a total of 30 cycles. The amplification condition for TGF $\beta 1$, type-I procollagen, type-III procollagen and GAPDH was pre-denaturation at $94^{\circ} \mathrm{C}$ for $5 \mathrm{~min}$, denaturation at $94^{\circ} \mathrm{C}$ for $30 \mathrm{~s}$, annealing at $55^{\circ} \mathrm{C}$ for $30 \mathrm{~s}$, and extension at $72{ }^{\circ} \mathrm{C}$ for $30 \mathrm{~s}$, a total of 28 cycles. The PCR products underwent electrophoresis and were scanned with a gel image analysis system (UVP, Upland, CA, USA). The intensity of TGF $\beta 1$, JNK, type-I and type-III procollagen was standardized to that of GAPDH mRNA.

\section{Western blot analyses}

The heart tissues of rats were lysed. Protein was extracted and measured using a bicinchoninic acid (BCA) Protein Assay Kit (Pierce, Rockford, IL, USA). Approximately $50 \mu \mathrm{g}$ of protein was separated by $10 \%$ sodium dodecyl sulfate-polyacrylamide gel electrophoresis (SDS-PAGE) and transferred to polyvinylidene fluoride (PVDF) membranes. The membranes were blocked with $5 \%$ fat-free milk in TBST buffer $(20 \mathrm{mmol} / \mathrm{L}$ Tris$\mathrm{HCl}$, pH 7.5, $150 \mathrm{mmol} / \mathrm{L} \mathrm{NaCl}$ and $0.05 \%$ Tween 20), and subsequently incubated with the following primary antibodies: anti-MYPT1, anti-JNK, and anti-TGF $\beta 1$ (Bioworld Technology, Minnesota, MN, USA); anti-phospho-MYPT1 (Thr ${ }^{853}$ ) and anti-phospho-JNK (T183/Y185) (Cell Signaling Technology, Danvers, MA, USA); anti-phospho-Smad2/3 (Ser $\left.{ }^{423 / 425}\right)$, anti-c-Jun and anti- $\beta$-actin (Santa Cruz Biotechnology) at $4{ }^{\circ} \mathrm{C}$ overnight. The mixture was washed and then incubated for $1 \mathrm{~h}$ with horseradish peroxidase (HRP)-conjugated secondary antibodies (KPL, Incorporated, Gaithersburg, MA, USA). The membranes were developed using ECL kit (Pierce). Quantification of bands was done by gel densitometry with a gel image analysis system (UVP). The phosphorylation level was normalized by total protein-band densitometry individually.

\section{Statistical analysis}

The SPSS 13.0 statistical software pack (SPSS, Chicago, IL, USA) was used for analysis. Data are mean \pm SD. All groups were tested for normal distribution and equal variance. Differences between two groups (at week 10) were assessed by the Student's $t$-test. Differences among three groups (at week 24) were assessed using one-way analysis of variance followed by the Student-Newman-Keuls' test if the $F$ value was significant. $P<0.05$ was considered statistically significant.

\section{Results}

\section{Insulin sensitivity and biochemical parameters}

Insulin sensitivity and biochemical parameters at week 10 are presented in Table 1. Diabetic rats had higher values of FBG, FINS, TG and BW compared with those in control rats, but there was no significant difference with respect to TC level. The GIR of diabetic rats was lower than that of control rats. These results suggested decreased insulin sensitivity and the development of insulin resistance in diabetic rats. That is, establishment of a model of type $2 \mathrm{DM}$ in rats was successful.

At week 24, compared with control rats, the levels of FBG, FINS, HbA1c, and HOMA-IR were significantly increased in untreated diabetic rats and fasudil-treated diabetic rats. There were no significant differences in these indices between untreated diabetic rats and treated diabetic rats, suggesting that $10 \mathrm{mg} \cdot \mathrm{kg}^{-1} \cdot \mathrm{d}^{-1}$ fasudil administered had no effects on glucose metabolism and insulin resistance (Table 2). Com-

Table 1. Effects of HFD+STZ on BW and biochemical parameters in rats at week 10. Mean \pm SD. ${ }^{c} P<0.01$ compared with control group.

\begin{tabular}{|c|c|c|c|c|c|c|c|}
\hline Group & $n$ & BW (g) & $\begin{array}{c}\mathrm{FBG} \\
(\mathrm{mmol} / \mathrm{L})\end{array}$ & $\begin{array}{l}\text { FINS } \\
(\mathrm{mU} / \mathrm{L})\end{array}$ & $\begin{array}{c}\text { GIR } \\
\left(\mathrm{mg} \cdot \mathrm{kg}^{-1} \cdot \mathrm{min}^{-1}\right)\end{array}$ & $\begin{array}{c}\mathrm{TG} \\
(\mathrm{mmol} / \mathrm{L})\end{array}$ & $\begin{array}{c}\mathrm{TC} \\
(\mathrm{mmol} / \mathrm{L})\end{array}$ \\
\hline Control & 6 & $276.65 \pm 33.18$ & $5.76 \pm 0.60$ & $51.88 \pm 11.40$ & $11.40 \pm 1.24$ & $0.26 \pm 0.07$ & $1.62 \pm 0.22$ \\
\hline
\end{tabular}

Differences between two groups were assessed by the Student's $t$-test. $n$, number of rats; BW, body weight; FBG, fasting blood glucose; FINS, fasting insulin; GIR, glucose infusion rate; TG, triglyceride; TC, total cholesterol. 
pared with control rats, TC and TG levels were significantly increased whereas BW was reduced in untreated diabetic rats and fasudil-treated diabetic rats. There were no significant differences in these indices between untreated diabetic rats and treated diabetic rats, suggesting that $10 \mathrm{mg} \cdot \mathrm{kg}^{-1} \cdot \mathrm{d}^{-1}$ fasudil administered did not affect lipid metabolism and body weight. Heart weight (HW) and the ratio of HW to BW (HW/BW) was significantly increased in untreated diabetic rats compared with control rats, there were no significant differences in HW and HW/BW between control rats and treated diabetic rats. There were no significant differences in SABP among the three groups (Table 3). These results suggested that diabetic rats incurred cardiac hypertrophy, and that fasudil treatment significantly suppressed the development of cardiac hypertrophy independent of blood pressure, blood lipid and glycemic control.

\section{Parameters of cardiac contractile function}

At week 24, compared with control rats, untreated diabetic hearts showed significantly reduced LVSP and $\pm \mathrm{d} p / \mathrm{d} t_{\max }$ whereas LVEDP was significantly increased. Treatment with fasudil significantly enhanced LVSP, $\pm \mathrm{d} p / \mathrm{d} t_{\max }$, and decreased LVEDP. There were no significant differences in HR among the three groups. The results indicated that fasudil effectively improved the contractile function of hearts from diabetic rats (Table 4).

\section{Immunohistochemistry and TEM study}

Representative patterns of Masson staining demonstrating cardiac fibrosis are shown in Figure 1A. The deposition of collagen in the myocardial interstitium from untreated diabetic hearts was significantly increased compared with that seen in control rats. However, treatment with fasudil significantly

Table 2. Effects of fasudil on glucose metabolism parameters in rats at week 24 . Mean \pm SD. ${ }^{c} P<0.01$ compared with control group.

\begin{tabular}{|c|c|c|c|c|c|}
\hline Group & $n$ & FBG (mmol/L) & FINS (mU/L) & HbA1c (\%) & HOMA-IR \\
\hline Control & 9 & $5.44 \pm 0.33$ & $54.83 \pm 11.74$ & $11.85 \pm 0.75$ & $13.70 \pm 3.91$ \\
\hline Diabetes & 8 & $20.48 \pm 3.12^{c}$ & $85.44 \pm 18.10^{\circ}$ & $19.38 \pm 3.30^{c}$ & $92.72 \pm 17.42^{\circ}$ \\
\hline DM+fas & 8 & $23.10 \pm 5.28$ & $83.10 \pm 13.14$ & $20.96 \pm 1.46$ & $81.43 \pm 12.81$ \\
\hline
\end{tabular}

Differences among three groups were assessed using one-way analysis of variance followed by the Student-Newman-Keuls' test if the $F$ value was significant. DM+fas: diabetic rats treated with fasudil; $n$, number of rats; FBG, fasting blood glucose; FINS, fasting insulin; HbA1c, hemoglobin A1c; HOMA-IR, homeostasis model assessment for insulin resistance, is calculated as FBG (mmol/L)×FINS $(\mathrm{mU} / \mathrm{L}) / 22.5$.

Table 3. Effects of fasudil on TG, TC, BW, HW, HW/BW, and SABP in rats at week 24 . Mean \pm SD. ${ }^{\mathrm{b}} P<0.05$, ${ }^{\mathrm{c}} P<0.01$ compared with control group. ${ }^{\mathrm{f}} P<0.01$ compared with diabetes group.

\begin{tabular}{|c|c|c|c|c|c|c|c|}
\hline Group & $n$ & $\mathrm{TG}(\mathrm{mmol} / \mathrm{L})$ & $\mathrm{TC}(\mathrm{mmol} / \mathrm{L})$ & BW (g) & HW (g) & $\mathrm{HW} / \mathrm{BW}\left(\times 10^{-3}\right)$ & $\mathrm{SABP}(\mathrm{mmHg})$ \\
\hline Control & 9 & $0.31 \pm 0.11$ & $1.69 \pm 0.35$ & $349.10 \pm 40.01$ & $0.91 \pm 0.06$ & $2.60 \pm 0.10$ & $119 \pm 4$ \\
\hline Diabetes & 8 & $2.07 \pm 0.28^{c}$ & $3.06 \pm 0.27^{c}$ & $297.06 \pm 35.1^{b}$ & $1.03 \pm 0.09^{c}$ & $3.48 \pm 0.17^{\circ}$ & $123 \pm 7$ \\
\hline $\mathrm{DM}+\mathrm{fas}$ & 8 & $2.02 \pm 0.56$ & $2.95 \pm 0.33$ & $301.77 \pm 37.39$ & $0.86 \pm 0.07^{f}$ & $2.67 \pm 0.14^{f}$ & $121 \pm 4$ \\
\hline
\end{tabular}

Differences among three groups were assessed using one-way analysis of variance followed by the Student-Newman-Keuls' test if the $F$ value was significant. DM+fas, diabetic rats treated with fasudil; $n$, number of rats; TG, triglyceride; TC, total cholesterol; BW, body weight; HW, heart weight; HW/ $\mathrm{BW}$, ratio of heart weight/body weight; SABP, systolic arterial blood pressure.

Table 4. Effects of fasudil on hemodynamics in rats at week 24 . Mean \pm SD. ${ }^{b} P<0.05,{ }^{c} P<0.01$ compared with control group. ${ }^{e} P<0.05$, ${ }^{f} P<0.01$ compared with diabetes group.

\begin{tabular}{|c|c|c|c|c|c|c|}
\hline Group & $n$ & HR (beat/min) & $\operatorname{LVSP}(\mathrm{mmHg})$ & LVEDP (mmHg) & $+\mathrm{dp} / \mathrm{d} t_{\max }(\mathrm{mmHg} / \mathrm{s})$ & $-\mathrm{dp} / \mathrm{d} t_{\max }(\mathrm{mmHg} / \mathrm{s})$ \\
\hline Control & 7 & $327 \pm 37$ & $139 \pm 21$ & $3.51 \pm 0.72$ & $6909 \pm 597$ & $6811 \pm 625$ \\
\hline Diabetes & 7 & $341 \pm 44$ & $101 \pm 17^{b}$ & $10.99 \pm 2.21^{c}$ & $3746 \pm 402^{c}$ & $3417 \pm 409^{c}$ \\
\hline $\mathrm{DM}+\mathrm{fas}$ & 6 & $337 \pm 42$ & $131 \pm 24^{\mathrm{e}}$ & $4.88 \pm 1.17^{f}$ & $6517 \pm 601^{f}$ & $5937 \pm 513^{f}$ \\
\hline
\end{tabular}

Differences among three groups were assessed using one-way analysis of variance followed by the Student-Newman-Keuls' test if the $F$ value was significant. DM+fas, diabetic rats treated with fasudil; $n$, number of rats; HR, heart rate; LVSP, left ventricular systolic pressure; LVEDP, left ventricular end-diastolic pressure; $\pm \mathrm{d} p / \mathrm{d} t_{\max }$, maximum change velocity of left ventricular pressure in the isovolumic contraction or relaxation period. 

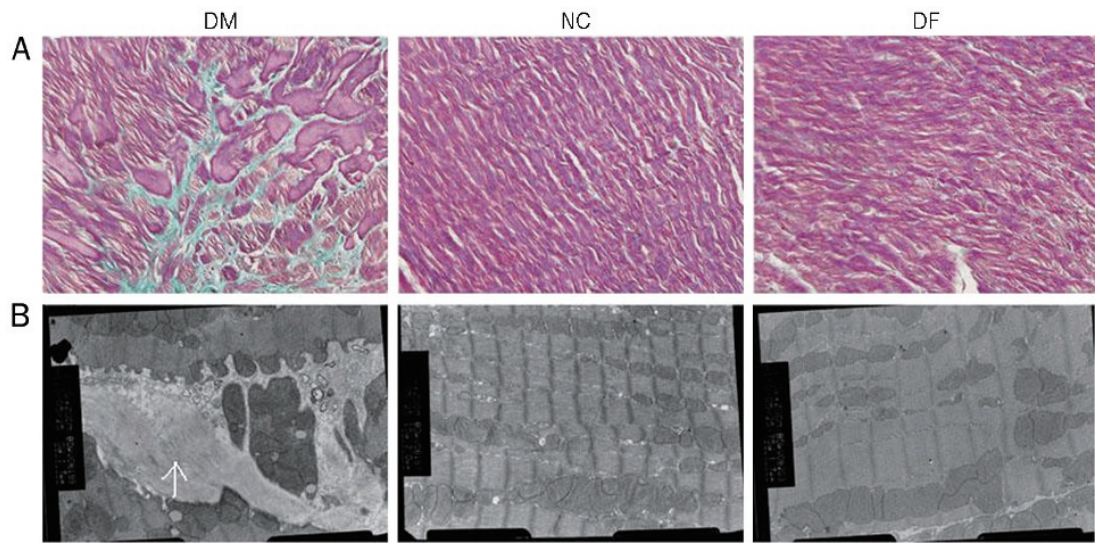

Figure 1. (A) Cardiac matrix deposition in Masson-stained sections of diabetic group (DM), control group (NC), and diabetic rats treated with fasudil group (DF). Heart tissues from the DM group show focal regions of fibrosis (blue) in the interstitium (collagen area $10.15 \% \pm 0.79 \%, P<0.01$ vs $N C$ : $2.42 \% \pm 0.33 \%$ and DF: $2.20 \% \pm 0.31 \%$ ). Magnification, $\times 400$. The number of rats in each group was six. (B) Representative electron micrograph obtained from the DM, NC, and DF. In diabetic rat hearts, "packages" of collagen fibrils (arrow) can also be seen between myocardiocytes (magnification, $\times 6000$ ). The control heart shows regular myofibrillar organization with evident $Z$ lines (magnification, $\times 5000$ ). The ultrastructural appearance of diabetic rats treated with fasudil is similar to that of control rats (magnification, $\times 6000$ ).

reduced the deposition of collagen. To identify collagen features in the cardiac fibrotic areas, SABC immunohistochemistry staining for type-I and type-III collagen was undertaken. An increase in deposition of type-I and type-III collagen was shown in untreated diabetic rats compared with that seen in control rats $(38.4 \% \pm 8.2 \%$ and $15.9 \% \pm 4.6 \%$ vs $11.2 \% \pm 2.9 \%$ and $4.3 \% \pm 1.5 \%$, respectively, $P<0.01)$. This increase was completely inhibited by treatment with fasudil $(13.7 \% \pm 4.1 \%$ and $5.1 \% \pm 2.3 \%$, respectively, $P<0.01$ vs $\mathrm{DM}$ ) (Figure $2 \mathrm{~A}$ and $2 \mathrm{~B}$ ).
The results of TEM observations showed the ultrastructure of the myocardium (Figure 1B). Untreated diabetic rats showed dense packages of collagen fibrils between cardiomyocytes, and large areas of cytoplasm had lost their regular myofibrillar organization. Control rats showed fibrils with $\mathrm{Z}$ lines regularly organized in the cardiomyocytes. The cardiac morphology of fasudil-treated diabetic rats was similar to that of control rats: regularly organized cytoplasm with numerous myofibrils arranged in parallel.
$\mathrm{DM}$

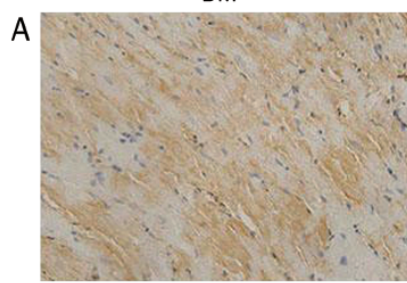

B
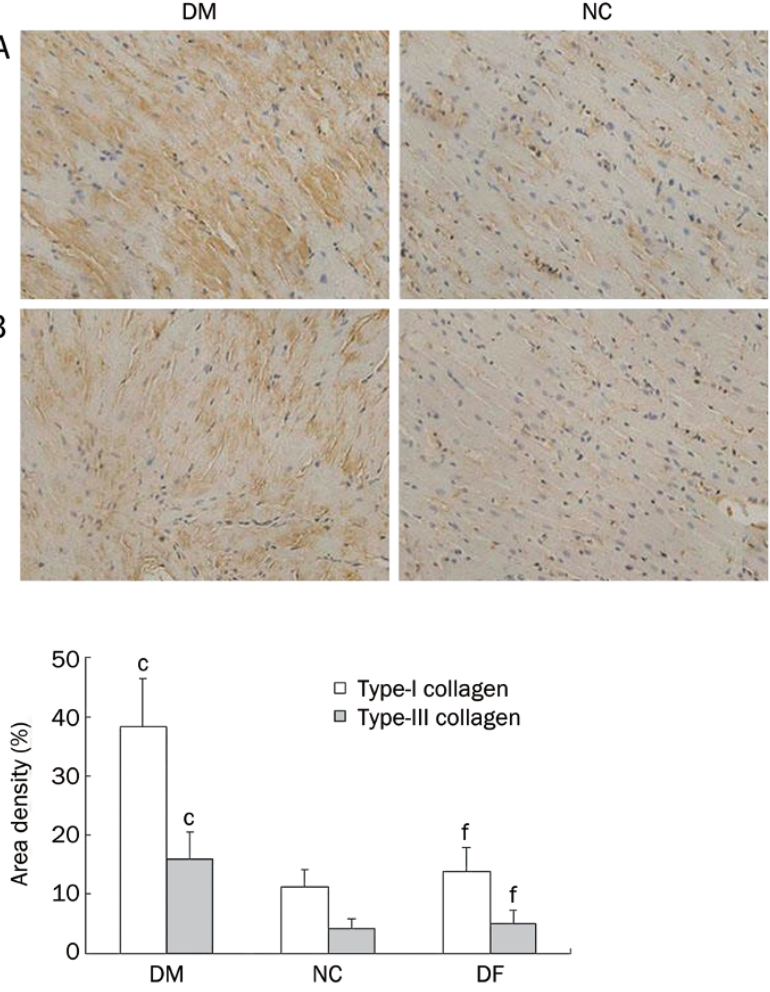

DF

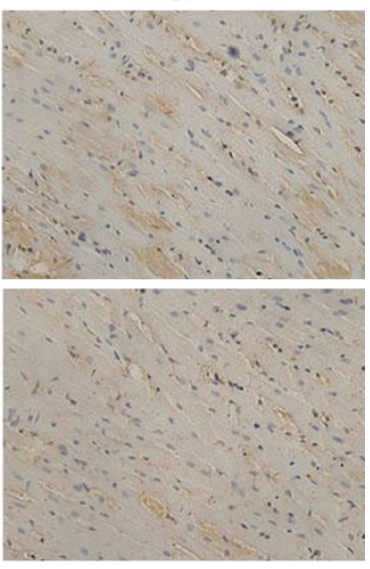

Figure 2. Immunostaining for type-I collagen (A) and type-III collagen (B) in the hearts of diabetic group (DM), control group (NC), and diabetic rats treated with fasudil group (DF). A brown color denotes positive staining. Diabetes is associated with increased immunostaining of type-I and type-III collagen in interstitial areas, whereas there is minimal immunostaining in control animals. Treatment of diabetic rats with fasudil was associated with a reduction in immunostaining of type-I and type-III collagen. Magnification, $\times 400$. The number of rats in each group was six. ${ }^{\mathrm{c}} P<0.01$ vs NC; ${ }^{\mathrm{f}} P<0.01$ vs DM. 


\section{Gene expression in LV tissues}

Compared with control rats, mRNA expression of type-I and type-III procollagen (Figure 3A and 3B) was significantly upregulated in hearts from untreated diabetic rats, but this expression was inhibited by treatment with fasudil. mRNA expression of JNK and TGF $\beta 1$ (Figure 3C and 3D) was significantly increased in the hearts of untreated diabetic rats; the upregulation of mRNA expression of JNK and TGF $\beta 1$ was blocked by treatment with fasudil. These results suggested that fasudil had inhibitory effects on DM-induced gene upregulation of type-I and type-III procollagen, JNK and TGF $\beta 1$.

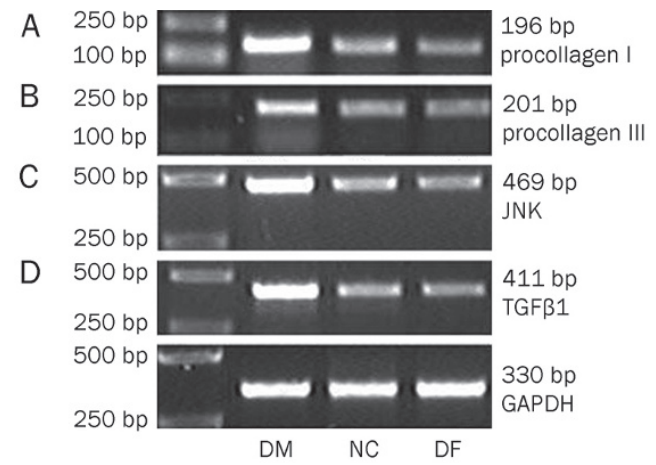

Figure 3. The mRNA expression of type-I procollagen (A), type-III procollagen (B), JNK (C), and TGFB1 (D) in diabetic rats (DM), control rats (NC) and diabetic rats treated with fasudil (DF) was evaluated by RTPCR. Intensity of type-I and type-III procollagen, JNK, and TGFß1 were standardized to that of GAPDH. The number of rats in each group was six. The mRNA expression of type-I, type-III procollagen, JNK, and TGFB1 were significantly increased in DM, $P<0.01$ vs NC. The gene expression was decreased in $\mathrm{DF}, P<0.01$ vs $\mathrm{DM}$.

\section{Protein expression in LV tissues}

ROCK can inhibit myosin phosphatase by phosphorylating myosin phosphatase target subunit-1 (MYPT1) ${ }^{[21]}$, so we measured MYPT1 phosphorylation as a maker of ROCK activity. The phosphorylation of MYPT1 was significantly increased in hearts from untreated diabetic rats, and this phosphorylation was inhibited by treatment with fasudil (Figure 4A). The phosphorylation of JNK (Figure 4B) and Smad2/3 (Figure $5 \mathrm{~A}$ ), as well as the protein levels of TGF $\beta 1$ (Figure 5B) and c-Jun (Figure 5C), were significantly enhanced in hearts from untreated diabetic rats, which were attenuated by treatment with fasudil. These results suggested that fasudil inhibited activation of the JNK and TGF $\beta 1 /$ Smad2/3 pathways.

\section{Discussion}

Several studies have reported that rats fed a HFD develop insulin resistance ${ }^{[22,23]}$. Low-dose STZ is known to induce a mild impairment of insulin secretion that is similar to a feature of late-stage type $2 \mathrm{DM}^{[16,24]}$. In the present study, at week 10 , the rats fed a HFD combined with low-dose STZ exhibited hyperglycemia, hyperlipidemia and hyperinsulinemia. The GIR of rats fed a HFD combined with low-dose STZ (as shown
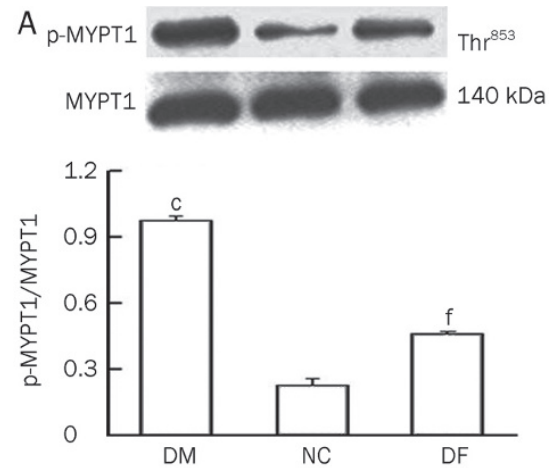

B
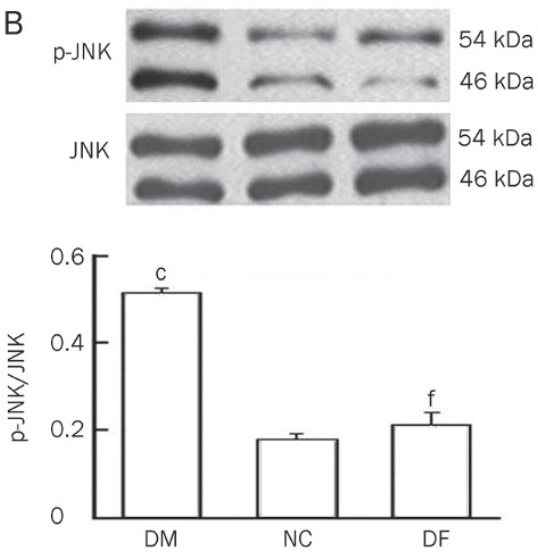

Figure 4. The phosphorylation of MYPT1 (A) and JNK (B) in diabetic rats (DM), control rats (NC), and diabetic rats treated with fasudil (DF) was measured by Western blot analyses. The activity of ROCK and JNK was expressed as p-MYPT1/MYPT1 and p-JNK/JNK, respectively. The number of rats in each group was six. ${ }^{c} P<0.01$ vs NC; ${ }^{f} P<0.01$ vs DM.

by the hyperinsulinemic-euglycemic clamp) was lower than that of control rats. Results suggested development of insulin resistance in rats fed a HFD combined with low-dose STZ. Hence, we had successfully established type 2 DM in rats, and this model would closely mimic the natural history and metabolic characteristics of type 2 DM in humans.

Long-term DM results in the development of diabetic cardiomyopathy independently of hypertension and coronary heart disease. Cardiac failure due to ventricular dysfunction is a characteristic of diabetic cardiomyopathy and occurs during the early stages of $\mathrm{DM}^{[25]}$. The present study revealed that hearts from diabetic rats showed significantly reduced contractility and prolonged diastole in vivo, which are hallmarks of diabetic cardiomyopathy. These findings are in agreement with other studies ${ }^{[26-28]}$. Consistent with their impaired cardiac function, diabetic rats developed myocardial fibrosis, the marked fibrotic regions (as observed by immunohistochemical staining), as well as deposition of collagen fibers in the interstitial spaces among cardiomyocytes (as observed by TEM), our observations are similar to those described in other reports $^{[29,30]}$. Increased myocardial fibrosis is a major factor responsible for myocardial stiffness and eventual systolic 
A
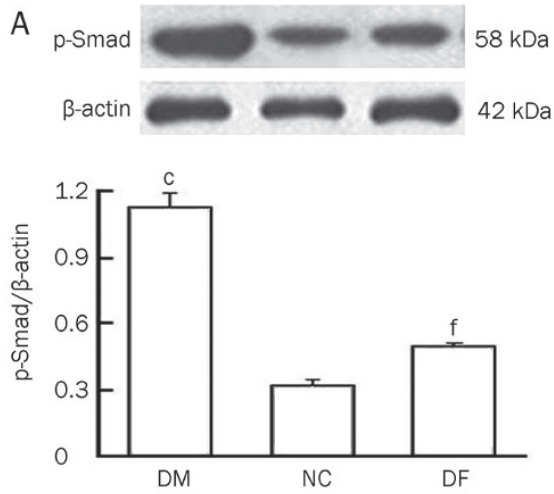

B
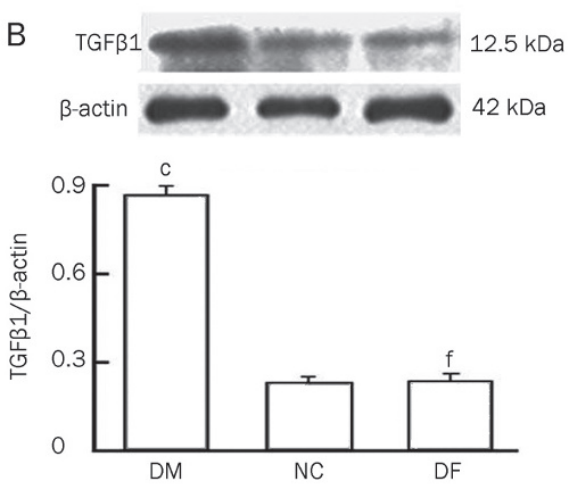

C
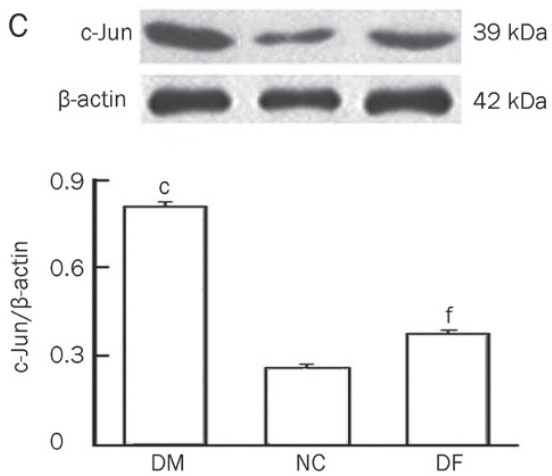

Figure 5. The phosphorylation of Smad2/3 (p-Smad2/3) (A), the protein levels of TGFB1 (B) and C-jun (C) in diabetic rats (DM), control rats (NC) and diabetic rats treated with fasudil (DF) were evaluated by Western blot analyses. p-Smad2/3, c-jun, and TGF $\beta 1$ protein levels were standardized respectively to that of $\beta$-actin as the internal control. The number of rats in each group was six. ${ }^{\mathrm{C}} P<0.01$ vs NC; ${ }^{\mathrm{f}} P<0.01$ vs DM.

dysfunction ${ }^{[31,32]}$. Our most significant finding is that treatment with fasudil attenuated interstitial myocardial fibrosis in diabetic rats and improved cardiac dysfunction. These effects were independent of blood pressure, blood lipid and glycemic control. The molecular mechanisms relating to the effects of fasudil on cardiac remodeling in diabetic hearts need to be elucidated.

RhoA/ROCK controls a wide variety of signal transduction pathways. ROCK exists as two isoforms: ROCK1 and ROCK2.
They share an overall homology of $92 \%$ in their kinase domains. Two major families of ROCK inhibitors, fasudil and Y-27632, are extensively used. They are non-isoform-selective ROCK inhibitors that target their ATP-dependent kinase domains and are therefore equipotent in terms of inhibiting both ROCK1 and ROCK2. Furthermore, at higher concentrations, these ROCK inhibitors could also inhibit other serinethreonine kinases, such as PKA and PKC ${ }^{[33]}$. Nevertheless, compared with the other kinases, fasudil and its active metabolite, hydroxyfasudil, are relatively more selective for ROCKs, with hydroxyfasudil being slightly more selective than fasudil and Y-27632 $2^{[33]}$. Fasudil is the only ROCK inhibitor practically available for long-term use in vivo. ROCK regulates not only the actin cytoskeleton but also the expression of genes associated with tissue fibrosis ${ }^{[10,34]}$. Data from some studies ${ }^{[35,36]}$ suggest diabetes impairs cardiac function through upregulation of RhoA. The increase in RhoA expression and activity in diabetic hearts resulted in increased phosphorylation of ROCK targets, LIM kinase and ezrin/radixin/moesin, leading to an increase in actin polymerization which might be associated with cardiac dysfunction. The inhibition of the RhoA/ ROCK pathway improved the contractile function of the diabetic heart. However, the precise mechanism is not fully clear behind upregulated RhoA/ROCK in diabetes. It has been suggested that RhoA activation regulates cardiac contractility and gene expression through the MAPK superfamily ${ }^{[2,37]}$. Recent studies $^{[12,38,39]}$ demonstrated that JNK is an important mediator of the fibrotic process. As a downstream target of JNK, c-Jun acts as transcription factor for numerous genes, and overexression of these genes is often associated with organ fibrosis. The present study explored the roles of Rho/ROCK and JNK pathways in myocardial fibrosis of diabetic cardiomyopathy, and results showed that hyperglycemia not only activated ROCK, but also upregulated the expression/activity of JNK and subsequent c-Jun protein levels. Fasudil not only inhibited ROCK activity but also repressed JNK over-activation in diabetic hearts, leading to a reduction in collagen production in hearts and an improvement in cardiac function. Moreover, the inhibitory effect of fasudil on collagen fibers was regulated at least as far upstream as the transcriptional level because treatment with fasudil suppressed the mRNA expression of type-I and type-III procollagen. The study demonstrated that activation of RhoA/ROCK was essential for myocardial fibrosis in diabetic rats. Fasudil could suppress myocardial fibrosis in diabetic rats, which might be associated with inhibition of JNK activation and subsequent expression of c-Jun. It has been reported that ROCK can directly activate JNK to stimulate c-Jun expression, the activity of which is independent of the ability of ROCK to promote actin polymerization ${ }^{[40]}$. Fasudil $\left(10-30 \mathrm{mg} \cdot \mathrm{kg}^{-1} \cdot \mathrm{d}^{-1}\right)$ can suppress renal interstitial fibrosis in insulin-resistant diabetic rats ${ }^{[7,8]}$. ROCK inhibition also improves pressure overload-induced cardiac hypertrophy and dysfunction ${ }^{[41]}$. Our findings are in agreement with the results of those studies ${ }^{[7,8,40,41]}$.

Collagen gene expression is also regulated by the Smad signaling pathway, which is activated by TGF $\beta^{[14,15]}$. ROCK plays 
a part in mediating renal fibrosis through a TGF $\beta$-dependent mechanism in vivo ${ }^{[9]}$. TGF $\beta$ activation in diabetic hearts led to increased production of collagen, indicating that the action of TGF $\beta$ also has an important role in cardiac fibrosis ${ }^{[15]}$. In the present study, in addition to examining TGF $\beta 1$ mRNA and protein expression, we also assessed its biological effects by evaluating one of its specific intracellular actions: phosphorylation of the TGF $\beta 1$ receptor-activated protein Smad2/3. The phosphorylation of Smad2/3 was increased in diabetic rat hearts, in tandem with increased TGF $\beta 1$ mRNA and protein expression. The results showed the increased production of TGF $\beta 1$ and activation of its signaling pathway in diabetic rats hearts. Treatment with fasudil led to a decrease in the level of TGF $\beta 1$ expression and phosphorylated Smad2/3. Our findings are consistent with the results of renal injury studies ${ }^{[9,42]}$. The study indicated that fasudil attenuated the augmentation actions of TGF $\beta 1$ in diabetic rats.

Insulin signaling is essential for normal glucose homeostasis. ROCK1 have been demonstrated as a novel regulator of glucose homeostasis and insulin sensitivity in vivo ${ }^{[43]}$. However, studies of the effects of ROCK inhibitors on glucose metabolism in vivo have yielded conflicting results. Kikuchi et $\mathrm{al}^{[7]}$ found that a low-dose of fasudil $\left(30 \mathrm{mg} \cdot \mathrm{kg}^{-1} \cdot \mathrm{d}^{-1}\right)$ did not affect glucose and lipid metabolism, but high-dose fasudil (100 $\mathrm{mg} \cdot \mathrm{kg}^{-1} \cdot \mathrm{d}^{-1}$ ) could ameliorate the metabolic disorder. Chronic treatment of obese $d b / d b$ mice with fasudil $\left(10 \mathrm{mg} \cdot \mathrm{kg}^{-1} \cdot \mathrm{d}^{-1}\right)$ was reported to have no effect on blood glucose levels and blood pressure $^{[8]}$. Differences that emerged among these studies may be correlated with the use of different inhibitors, doses, treatment times and animal models. In present study, a low-dose of fasudil $\left(10 \mathrm{mg} \cdot \mathrm{kg}^{-1} \cdot \mathrm{d}^{-1}\right)$ have no effects on BG, insulin resistance and BW, as well as lipid metabolism and blood pressure in diabetic rats. The cardiac protective effects of fasudil were independent of blood pressure and glycemic control.

The present study provides evidence that RhoA/ROCK, $\mathrm{JNK}$, and TGF $\beta /$ Smad pathways are involved in myocardial fibrosis in rats with type 2 diabetes. JNK and Smad2/3 may be the downstream signaling molecule of the RhoA/ROCK pathway. It is plausible that the RhoA/ROCK signaling pathway is associated with nuclear signal transduction at least in part through the JNK and TGF $\beta /$ Smad pathways, which regulates the expression of genes and proteins that are pertinent to myocardial fibrosis. Our current findings should shed some light on a better understanding of the role of RhoA/ ROCK pathway in the pathogenesis of diabetic cardiomyopathy. However, there were some experimental limitations in our study. First, to better understand the mechanism of action involved, cell culture study is needed to verify the causeeffect relationship with regards to the role of these signaling molecules in diabetic cardiac fibrosis. Second, it is known that diabetic cardiomyopathy is associated with significant increased oxidative stress which could lead to JNK activation and increased TGF $\beta^{[29,44]}$, so the contents of oxidative stress in the heart should be described. Third, there are only small number of rats in the study, this maybe limits statistical power and their results.
Treatments used to prevent the development/progression of diabetic cardiomyopathy are not fully effective. Fasudil is the only ROCK inhibitor approved for human use. Importantly, several studies have documented the beneficial effects of ROCK inhibitors in patients with cardiovascular diseases and cerebral vasospasm ${ }^{[6,45-47]}$. The present study suggests that Rho/ROCK is substantially involved in remodeling of the myocardial matrix in diabetic rats, which may be associated with activation of the JNK pathway and TGF $\beta /$ Smad pathway. Fasudil suppresses myocardial fibrosis in diabetic rats at least in part by inhibiting activation of JNK and TGF $\beta /$ Smad, and these beneficial structural effects of fasudil are independent of blood pressure and glycemic control. Inhibition of the Rho/ROCK pathway may be a novel therapeutic target for prevention of diabetic cardiomyopathy.

\section{Acknowledgements}

This research was supported by a grant from Hebei Province Department of Health, Hebei, China (a principal item, grant number 20090007). The authors thank Kai-xia ZHANG, Chunfang ZHAO and Rong ZHANG MD for their technical assistance.

\section{Author contribution}

Yong-jun LI and Hong ZHOU designed research; Zhan-sheng ZHAO, Hong ZHOU, Mian WANG, and Feng-ling MENG performed research; Rui-ying WANG, Yong-gui DENG, Bingyan GUO, and Li-hui ZHANG analyzed data; and Hong ZHOU and Yong-jun LI wrote the paper.

\section{References}

1 Gustafsson I, Hildebrandt P. Early failure of the diabetic heart. Diabetes Care 2001; 24: 3-4.

2 Poirier P, Bogaty P, Garneau C, Marois L, Dumesnil JG. Diastolic dysfunction in normotensive men with well-controlled type 2 diabetes: importance of maneuvers in echocardiographic screening for preclinical diabetic cardiomyopathy. Diabetes Care 2001; 24: 5-10.

3 Riento K, Ridley AJ. Rocks: multifunctional kinases in cell behaviour. Nat Rev Mol Cell Biol 2003; 4: 446-56.

4 Rikitake Y, Liao JK. Rho GTPases, statins, and nitric oxide. Circ Res 2005; 97: 1232-5.

5 Masumoto A, Hirooka Y, Shimokawa H, Hironaga K, Setoguchi S, Takeshita A. Possible involvement of Rho-kinase in the pathogenesis of hypertension in humans. Hypertension 2001; 38: 1307-10.

6 Shibuya M, Hirai S, Seto M, Satoh S, Ohtomo E, Fasudil Ischemic Stroke Study Group. Effects of fasudil in acute ischemic stroke: results of a prospective placebo-controlled double-blind trial. J Neurol Sci 2005; 238: 31-9.

7 Kikuchi Y, Yamada M, Imakiire T, Kushiyama T, Higashi K, Hyodo N, et al. A Rho-kinase inhibitor, fasudil, prevents development of diabetes and nephropathy in insulin-resistant diabetic rats. J Endocrinol 2007; 192: 595-603.

8 Kolavennu V, Zeng L, Peng H, Wang Y, Danesh FR. Targeting of RhoA/ ROCK signaling ameliorates progression of diabetic nephropathy independent of glucose control. Diabetes 2008; 57: 714-23.

9 Nishikimi T, Akimoto K, Wang X, Mori Y, Tadokoro K, Ishikawa Y, et al. Fasudil, a Rho-kinase inhibitor, attenuates glomerulosclerosis in Dahl salt-sensitive rats. J Hypertens 2004; 22: 1787-96. 
10 Wang YX, da Cunha V, Martin-McNulty B, Vincelette J, Li W, Choy DF, et al. Inhibition of Rho-kinase by fasudil attenuated angiotensin IIinduced cardiac hypertrophy in apolipoprotein $\mathrm{E}$ deficient mice. Eur J Pharmacol 2005; 512: 215-22.

11 Omura T, Yoshiyama M, Matsumoto R, Kusuyama T, Enomoto S, Nishiya D, et al. Role of c-Jun $\mathrm{NH}_{2}$-terminal kinase in G-proteincoupled receptor agonist-induced cardiac plasminogen activator inhibitor-1 expression. J Mol Cell Cardiol 2005; 38: 583-92.

12 Hao GH, Niu XL, Gao DF, Wei J, Wang NP. Agonists at PPAR-gamma suppress angiotensin II-induced production of plasminogen activator inhibitor-1 and extracellular matrix in rat cardiac fibroblasts. $\mathrm{Br} \mathrm{J}$ Pharmacol 2008; 153: 1409-19.

13 Ohtsu H, Mifune M, Frank GD, Saito S, Inagami T, Kim-Mitsuyama S, et al. Signal-crosstalk between Rho/ROCK and c-Jun $\mathrm{NH}_{2}$-terminal kinase mediates migration of vascular smooth muscle cells stimulated by angiotensin II. Arterioscler Thromb Vasc Biol 2005; 25: 1831-6.

14 Zyadeh FN, Sharma K, Erickson M, Wolf G. Stimulation of collagen gene expression and protein synthesis in murine mesangial cells by high glucose in mediated by autocrine activation of transforming growth factor- $\beta$. J Clin Invest 1994; 93: 536-42.

15 Martin J, Kelly DJ, Mifsud SA, Zhang Y, Cox AJ, See F, et al. Tranilast attenuates cardiac matrix deposition in experimental diabetes: role of transforming growth factor $\beta$. Cardiovasc Res 2005; 65: 694-701.

16 Srinivasan K, Viswanad B, Asrat L, Kaul CL, Ramarao P. Combination of high-fat diet-fed and low-dose streptozotocin-treated rat: a model for type 2 diabetes and pharmacological screening. Pharmacol Res 2005; 52: 313-20.

17 Islam MS, Choi H. Nongenetic model of type 2 diabetes: a comparative study. Pharmacology 2007; 79: 243-9.

18 Matthews DR, Hosker JP, Rudenski AS, Naylor BA, Treacher DF, Turner RC. Homeostasis model assessment: insulin resistance and beta-cell function from fasting plasma glucose and insulin concentrations in man. Diabetologia 1985; 28: 412-9.

19 Tran TT, Gupta N, Goh T, Naigamwalla D, Chia MC, Koohestani N, et al. Direct measure of insulin sensitivity with the hyperinsulinemiceuglycemic clamp and surrogate measures of insulin sensitivity with the oral glucose tolerance test: correlations with aberrant crypt foci promotion in rats. Cancer Epidemiol Biomarkers Prev 2003; 12 : 47-56.

20 Tzanidis A, Hannan RD, Thomas WG, Onan D, Autelitano DJ, See F, et al. Direct actions of urotensin II on the heart: implications for cardiac fibrosis and hypertrophy. Circ Res 2003; 93: 246-53.

21 Wakino S, Hayashi K, Kanda T, Tatematsu S, Homma K, Yoshioka K, et al. Peroxisome proliferator-activated receptor gamma ligands inhibit Rho/Rho kinase pathway by inducing protein tyrosine phosphatase SHP-2. Circ Res 2004; 95: e45-55.

22 Tanaka S, Hayashi T, Toyoda T, Hamada T, Shimizu Y, Hirata M, et al. High-fat diet impairs the effects of a single bout of endurance exercise on glucose transport and insulin sensitivity in rat skeletal muscle. Metabolism 2007; 56: 1719-28

23 Flanagan AM, Brown JL, Santiago CA, Aad PY, Spicer LJ, Spicer MT. High-fat diets promote insulin resistance through cytokine gene expression in growing female rats. J Nutr Biochem 2008; 19: 50513.

24 Reed MJ, Meszaros K, Entes LJ, Claypool MD, Pinkett JG, Gadbois TM, et al. A new rat model of type 2 diabetes: the fat-fed, streptozotocintreated rat. Metabolism 2000; 49: 1390-4.

25 Redfield MM, Jacobsen SJ, Burnett JC Jr, Mahoney DW, Bailey KR, Rodeheffer RJ. Burden of systolic and diastolic ventricular dysfunction in the community: appreciating the scope of the heart failure epidemic. JAMA 2003; 289: 194-202.
26 Vadlamudi RV, Rodgers RL, McNeill JH. The effect of chronic alloxanand streptozotocin-induced diabetes on isolated rat heart performance. Can J Physiol Pharm 1982; 60: 902-11.

27 Norby FL, Aberle NS, Kajstura J, Anversa P, Ren J. Transgenic overexpression of insulin-like growth factor I prevents streptozotocininduced cardiac contractile dysfunction and beta-adrenergic response in ventricular myocytes. J Endocrinol 2004; 180: 175-82.

28 Ren J, Duan J, Thomas DP, Yang X, Sreejayan N, Sowers JR, et al. IGF-I alleviates diabetes-induced RhoA activation, eNOS uncoupling, and myocardial dysfunction. Am J Physiol Regul Integr Comp Physiol 2008; 294: R793-802.

29 Aragno M, Mastrocola R, Alloatti G, Vercellinatto I, Bardini P, Geuna S, et al. Oxidative stress triggers cardiac fibrosis in the heart of diabetic rats. Endocrinology 2008; 149: 380-8.

30 Jin D, Takai S, Sugiyama T, Hayashi T, Fukumoto M, Oku H, et al. Longterm angiotensin II blockade may improve not only hyperglycemia but also age-associated cardiac fibrosis. J Pharmacol Sci 2009; 109: 275-84.

31 Yamamoto K, Masuyama T, Sakata Y, Nishikawa N, Mano T, Yoshida $\mathrm{J}$, et al. Myocardial stiffness is determined by ventricular fibrosis, but not by compensatory or excessive hypertrophy in hypertensive heart. Cardiovasc Res 2002; 55: 76-82.

32 Kai H, Kuwahara F, Tokuda K, Imaizumi T. Diastolic dysfunction in hypertensive hearts: roles of perivascular inflammation and reactive myocardial fibrosis. Hypertens Res 2005; 28: 483-90.

33 Rikitake Y, Kim HH, Huang Z, Seto M, Yano K, Asano T, et al. Inhibition of Rho kinase (ROCK) leads to increased cerebral blood flow and stroke protection. Stroke 2005; 36: 2251-7.

34 Fukushima M, Nakamuta M, Kohjima M, Kotoh K, Enjoji M, Kobayashi $\mathrm{N}$, et al. Fasudil hydrochloride hydrate, a Rho-kinase (ROCK) inhibitor, suppresses collagen production and enhances collagenase activity in hepatic stellate cells. Liver Int 2005; 25: 829-38.

35 Lin G, Craig GP, Zhang L, Yuen VG, Allard M, McNeill JH, et al. Acute inhibition of Rho-kinase improves cardiac contractile function in streptozotocin-diabetic rats. Cardiovasc Res 2007; 75: 51-8.

36 Soliman H, Craig GP, Nagareddy P, Yuen VG, Lin G, Kumar U, et al. Role of inducible nitric oxide synthase in induction of RhoA expression in hearts from diabetic rats. Cardiovasc Res 2008; 79: 322-30.

37 Kobayashi N, Horinaka S, Mita S, Nakano S, Honda T, Yoshida K, et al. Critical role of Rho-kinase pathway for cardiac performance and remodeling in failing rat hearts. Cardiovasc Res 2002; 55: 757-67.

38 Chen X, Tian Y, Yao L, Zhang J, Liu Y. Hypoxia stimulates proliferation of rat neural stem cells with influence on the expression of cyclin $\mathrm{D} 1$ and c-Jun $\mathrm{N}$-terminal protein kinase signaling pathway in vitro. Neuroscience 2010; 165: 705-14.

39 Zhou $\mathrm{CH}$, Wu XH, Wu YQ. Nobiletin, a dietary phytochemical, inhibits vascular smooth muscle cells proliferation via calcium-mediated c-Jun N-terminal kinases pathway. Eur J Pharmacol 2009; 615: 55-60.

40 Marinissen MJ, Chiariello M, Tanos T, Bernard O, Narumiya S, Gutkind JS. The small GTP-binding protein RhoA regulates c-jun by a ROCKJNK signaling axis. Mol Cell 2004; 14: 29-41.

41 Phrommintikul A, Tran L, Kompa A, Wang B, Adrahtas A, Cantwell D, et al. Effects of a Rho kinase inhibitor on pressure overload induced cardiac hypertrophy and associated diastolic dysfunction. Am J Physiol Heart Circ Physiol 2008; 294: H1804-14.

42 Sun GP, Kohno M, Guo P, Nagai Y, Miyata K, Fan YY, et al. Involvements of Rho-kinase and TGF-beta pathways in aldosterone-induced renal injury. J Am Soc Nephrol 2006; 17: 2193-201.

43 Lee DH, Shi J, Jeoung NH, Kim MS, Zabolotny JM, Lee SW, et al. Targeted disruption of ROCK1 causes insulin resistance in vivo. J Biol Chem 2009; 284: 11776-80. 
44 Han MJ, Kim BY, Yoon So, Chung AS. Cell proliferation induced by reactive oxygen species is mediated via mitogen-activated protein kinase in Chinese hamster lung fibroblast (V79) cells. Mol Cells 2003; 15: 94-101.

45 Kishi T, Hirooka Y, Masumoto A, Ito K, Kimura Y, Inokuchi K, et al. Rho-kinase inhibitor improves increased vascular resistance and impaired vasodilation of the forearm in patients with heart failure. Circulation 2005; 111: 2741-7.
46 Masumoto A, Mohri M, Shimokawa H, Urakami L, Usui M, Takeshita A. Suppression of coronary artery spasm by the Rho-kinase inhibitor fasudil in patients with vasospastic angina. Circulation 2002; 105 : 1545-7.

47 Shimokawa H, Hiramori K, linuma H, Hosoda S, Kishida H, Osada H, et al. Anti-anginal effect of fasudil, a Rho-kinase inhibitor, in patients with stable effort angina: a multicenter study. J Cardiovasc Pharmacol 2002; 40: 751-61. 